\title{
Decay Rate for a Viscoelastic Equation with Strong Damping and Acoustic Boundary Conditions
}

\author{
Zhiyong Ma \\ College of Science, Shanghai Second Polytechnic University, Shanghai, China \\ Email: mazhiyong1980@hotmail.com
}

How to cite this paper: Ma, Z.Y. (2017) Decay Rate for a Viscoelastic Equation with Strong Damping and Acoustic Boundary Conditions. Journal of Applied Mathematics and Physics, 5, 922-932.

https://doi.org/10.4236/jamp.2017.54081

Received: February 13, 2017

Accepted: April 27, 2017

Published: April 30, 2017

Copyright $\odot 2017$ by author and Scientific Research Publishing Inc. This work is licensed under the Creative Commons Attribution International License (CC BY 4.0).

http://creativecommons.org/licenses/by/4.0/

\section{c) (†) Open Access}

\begin{abstract}
This paper is concerned with a nonlinear viscoelastic equation with strong damping: $\left|u_{t}\right|^{\rho} u_{t t}-\Delta u-\Delta u_{t t}+\int_{0}^{t} g(t-s) \Delta u(x, s) \mathrm{d} s-\Delta u_{t}=0$,. The objective of the present paper is to provide some results on the long-time behavior to this equation with acoustic boundary conditions. By using the assumptions on the relaxation function due to Tatar [1], we show an arbitrary rate of decay with not necessary of an exponential or polynomial one and without the assumption $\int_{0}^{\infty} g(s) \mathrm{d} s<\frac{1}{2}$ condition. The result extends and improves some results given in Cavalcanti [2].
\end{abstract}

\section{Keywords}

Viscoelastic Equation, Decay Rate, Acoustic Boundary Condition

\section{Introduction}

In this paper, we investigate the following viscoelastic system with acoustic boundary conditons

$$
\begin{gathered}
\left|u_{t}\right|^{\rho} u_{t t}-\Delta u-\Delta u_{t t}+\int_{0}^{t} g(t-s) \Delta u(x, s) \mathrm{d} s-\Delta u_{t}=0, \quad(x, t) \in(0,+\infty), \\
\frac{\partial u_{t}}{\partial v}(x, t)=0 \quad(x, t) \in \Gamma \times[0,+\infty), \\
u(x, t)=0, \quad(x, t) \in \Gamma_{1} \times[0,+\infty), \\
\frac{\partial u_{t t}}{\partial v}(x, t)+\frac{\partial u}{\partial v}(x, t)-\int_{0}^{t} g(t-s) \frac{\partial u}{\partial v}(x, s) \mathrm{d} s=y_{t} \quad(x, t) \in \Gamma_{0} \times[0,+\infty), \\
u_{t}(x, t)+p(x) y_{t}+q(x) y(x, t)=0 \quad(x, t) \in \Gamma_{0} \times[0,+\infty),
\end{gathered}
$$




$$
u(x, 0)=u_{0}(x), u_{t}(x, 0)=u_{1}(x), \quad x \in \Omega,
$$

where $\Omega \subseteq \mathbb{R}^{n}(n=1,2)$ is a bounded domain with smooth boundary $\Gamma=\Gamma_{0} \bigcup \Gamma_{1}, v$ is the unit outward normal to $\Gamma$, the function $g$ represents the kernel of a memory, $p$ and $q$ are specific functions, and $\rho$ is a real number such that

$$
1<\rho \leq \frac{2}{n-2} \text { if } n \geq 3 ; \quad \rho>1 \text { if } n=1,2 .
$$

Our problem is of the form

$$
f\left(u_{t}\right) u_{t t}-\Delta u-\Delta u_{t t}=0
$$

which has several modeling features. In the case, $f\left(u_{t}\right)$ is a constant; Equation (8) has been used to model extensional vibrations of thin rods (see Love [3], Chapter 20). In the case, $f\left(u_{t}\right)$ is not a constant; Equation (8) can model materials whose density depends on the velocity $u_{t}$, for instance, a thin rod which possesses a rigid surface and with an interior which can deform slightly. We refer the reader to Fabrizio and Morro [4] for several other related models.

Recently, Liu [5] considered the following viscoelastic problem with acoustic boundary conditions

$$
\begin{gathered}
u_{t t}-\Delta u+\int_{0}^{t} g(t-s) \Delta u(x, s) \mathrm{d} s=0, \quad(x, t) \in(0,+\infty), \\
u(x, t)=0, \quad(x, t) \in \Gamma_{1} \times[0,+\infty), \\
\frac{\partial u}{\partial v}(x, t)-\int_{0}^{t} g(t-s) \frac{\partial u}{\partial v}(x, s) \mathrm{d} s=y_{t} \quad(x, t) \in \Gamma_{0} \times[0,+\infty), \\
u_{t}(x, t)+p(x) y_{t}+q(x) y(x, t)=0 \quad(x, t) \in \Gamma_{0} \times[0,+\infty), \\
u(x, 0)=u_{0}(x), u_{t}(x, 0)=u_{1}(x), \quad x \in \Omega,
\end{gathered}
$$

the authors obtain an arbitrary decay rate of the energy. In the pioneering paper [6], Beale and Rosencrans considered the acoustic boundary condition (1.12) and the coupled impenetrability boundary condition (1.11) with a general form, which had the presence of $y_{t t}$ in (1.2), in a study of the model for acoustic wave motion of a fluid interacting with a so-called locally reacting surface. Recently, many authors treated wave equations with acoustic boundary conditions, see [7] [8] [9] [10] and references therein. For instance, Rivera and Qin [10] proved the polynomial decay for the wave motion with general acoustic boundary conditions by using the Lyapunov functional technique. Frota and Larkin [8] established global solvability and the exponential decay for problems (1.9)-(1.13) with $g \equiv 0$. They overcame the difficulties which were arisen due to the absence of $y_{t t}$ in (1.12) by using the degenerated second order equation. Recently, Park and Park [9] investigated problems (1.9)-(1.13) and proved general rates of decay which depended on the behavior of $g$, under the additional assumption of that $\int_{0}^{+\infty} g(s) \mathrm{d} s$.

Many authors have focused on the viscoelastic problem. In the pioneer work of Dafermos [11] [12], existence and asymptotic stability for a one-dimensional vis- 
coelastic problem were proved but no rate of decay has been specified. Since then problems related to viscoelasticity have attracted a great deal of attention [13] [14] [15]. It seems all started with kernels of the form $g(t)=e^{-\beta t}, \beta>0$, then with kernels satisfying $-\xi_{1} g(t) \leq g^{\prime}(t) \leq-\xi_{2} g(t)$, for all $t \geq 0$, for some constants $\xi_{1}$ and $\xi_{2}$ and some other conditions on the second derivative, Cavalcanti et al. [2] studied the following equation with Dirichlet boundary conditions

$$
\left|u_{t}\right|^{\rho} u_{t t}-\Delta u-\Delta u_{t t}+g * \Delta u-\gamma \Delta u_{t}=0
$$

where $g * \Delta u=\int_{0}^{t} g(t-s) \Delta u(s) \mathrm{d} s$. They established a global existence result for $\gamma \geq 0$ and an exponential decay of energy for $\gamma>0$, and studied the interaction within the $\left|u_{t}\right|^{\rho} u_{t t}$ and the memory term $g * \Delta u$. Messaoudi and Tatar [16] established, for small initial data, the global existence and uniform stability of solutions to the equation

$$
\left|u_{t}\right|^{\rho} u_{t t}-\Delta u-\Delta u_{t t}+g * \Delta u=b|u|^{p-2} u
$$

with Dirichlet boundary condition, where $\gamma \geq 0, \rho, b>0, p>2$ are constants. In the case $b=0$ in (15), Messaoudi and Tatar [17] proved the exponential decay of global solutions to (15) without smallness of initial data, considering only the dissipation effect given by the memory.

In [18] [19], the condition has been replaced by $g^{\prime}(t) \leq-\xi(t) g(t)$, where $\xi(t)$ is a positive function. Similarly, Han and Wang [20] proved the energy decay for the viscoelastic equation with nonlinear damping

$$
\left|u_{t}\right|^{\rho} u_{t t}-\Delta u-\Delta u_{t t}+g * \Delta u+\left|u_{t}\right|^{m} u_{t}=0,
$$

with Dirichlet boundary condition, where $\rho>0, m>0$ are constants. Then Park and Park [21] established the general decay for the viscoelastic problem with nonlinear weak damping

$$
\left|u_{t}\right|^{\rho} u_{t t}-\Delta u_{t t}-\Delta u+g * \Delta u+h\left(u_{t}\right)=0,
$$

with the Dirichlet boundary condition, where $\rho>0$ is a constant. We also mention that Fabrizio and Polidoro [22] obtained the exponential decay result under the conditions that $g^{\prime}(t) \leq 0$ and $e^{\alpha t} g(t) \in L^{1}(0,+\infty)$ for some $\alpha>0$. Recently, Tatar [23] improved these results by removing the last condition and established a polynomial asymptotic stability. In fact, he considered the kernels having small flat zones and these zones are not too big (see also [24] for the case of coupled system). More recently, under the assumptions that $g^{\prime}(t) \leq 0$ and $g(t) \gamma(t) \in L^{1}(0,+\infty)$ for some nonnegative function $\gamma(t)$, Tatar [1] generalized these works to an arbitrary decay for wave equation with a viscoelastic damping term. Moreover, we would like to mention some results in [25]-[30].

The rest of our paper is organized as follows. In Section 2, we give some preparations for our consideration and our main result. The statements and the proofs of our main results will be given in Section 3.

For convenience, we denote the norm and scalar product in $L^{2}(\Omega)$ by $\|$. and $(\cdot, \cdot)$, respectively. $C$ denotes a general positive constant, which may be different in different estimates. 


\section{Preliminaries and Main Result}

For the memory kernel $g$ we assume that:

$\left(H_{1}\right) g: \mathbb{R}^{+} \rightarrow \mathbb{R}^{+}$is a non-increasing differentiable function satisfying that

$$
g(0)>0, l=1-\int_{0}^{+\infty} g(s) \mathrm{d} s>0 .
$$

$\left(H_{2}\right)$ suppose that there exists a nondecreasing function $\gamma(t)>0$ such that $\frac{\gamma^{\prime}(t)}{\gamma(t)}=\eta(t)$ is a decreasing function and $\int_{0}^{+\infty} g(s) \gamma(s) \mathrm{d} s<+\infty$.

For the functions $p$ and $q$, we assume that $p, q \in C\left(\Gamma_{0}\right)$ and $p(x)>0$ and $q(x)>0$ for all $x \in \Gamma_{0}$. This assumption implies that there exist positive constants $p_{i}, q_{i}(i=0,1)$ such that

$$
p_{0} \leq p(x) \leq p_{1}, \quad q_{0} \leq q(x) \leq q_{1}, x \in \Gamma_{0} .
$$

We use the notation

$$
\begin{aligned}
& V=\left\{u \in H^{1}(\Omega): u=0 \text { on } \Gamma_{1}\right\}, \\
& (u, v)=\int_{\Omega} u(x) v(x) \mathrm{d} x, \text { and }(u, v)_{\Gamma_{0}}=\int_{\Gamma_{0}} u(x) v(x) \mathrm{d} \Gamma .
\end{aligned}
$$

Let $\lambda$ and $\tilde{\lambda}$ be the smallest positive constants such that

$$
\|u\|^{2} \leq \lambda\|\nabla u\|^{2}, \quad\|u\|_{\Gamma_{0}}^{2} \leq \tilde{\lambda}\|\nabla u\|^{2} .
$$

Firstly, we have the following existence and uniqueness results, it can be established by adopting the arguments of [2] [31].

Theorem 2.1 Let $\left(u_{0}, u_{1}\right) \in\left(V \bigcap H^{2}(\Omega)\right) \times V$. Assume that $H_{1}, H_{2}$ and (2.2) hold. There exists a unique pair of functions $\left(u, y_{t}\right)$, which is a solution to the problem (1.1) in the class

$$
\begin{aligned}
& u \in L^{\infty}\left(0, T, V \cap H^{2}(\Omega)\right), \quad u_{t} \in L^{\infty}(0, T, V), \\
& u_{t t} \in L^{\infty}\left(0, T, L^{2}(\Omega)\right), \quad y, y^{t} \in L^{2}\left(\mathbb{R}^{+} ; L^{2}\left(\Gamma_{0}\right)\right) .
\end{aligned}
$$

We introduce the modified energy functional

$$
\begin{aligned}
E(t)= & \frac{1}{\rho+2}\left\|u_{t}\right\|_{\rho+2}^{\rho+2}+\frac{1}{2}\left(1-\int_{0}^{t} g(s) \mathrm{d} s\right)\|\nabla u\|^{2}+\frac{1}{2}(g \circ \nabla u)(t) \\
& +\frac{1}{2}\left\|\nabla u_{t}(t)\right\|^{2}+\frac{1}{2} \int_{\Gamma_{0}} q(x)|y(x, t)|^{2} \mathrm{~d} \Gamma,
\end{aligned}
$$

where

$$
(g \circ \nabla u)(t)=\int_{0}^{t} g(t-s)\|\nabla u(t)-\nabla u(s)\|^{2} \mathrm{~d} s .
$$

Clearly

$$
\frac{\mathrm{d}}{\mathrm{d} t} E(t)=-\left\|\nabla u_{t}(t)\right\|^{2}-\frac{1}{2} g(t)\|\nabla u\|^{2}+\frac{1}{2}\left(g^{\prime} \circ \nabla u\right)-\int_{\Gamma_{0}} p y_{t}^{2} .
$$

To state our main result, we introduce the following notations as in [32]. For every measurable set $\mathcal{A} \subset \mathbb{R}^{+}$, we define the probability measure $\hat{g}$ by

$$
\hat{g}(\mathcal{A})=\frac{1}{1-l} \int_{\mathcal{A}} g(s) \mathrm{d} s .
$$


The flatness set and the flatness rate of $g$ are defined by

$$
\mathcal{F}_{g}=\left\{s \in \mathbb{R}^{+}: g(s)>0 \text { and } g^{\prime}(s)=0\right\}
$$

and

$$
\mathcal{R}_{g}=\hat{g}\left(\mathcal{F}_{g}\right)=\frac{1}{1-l} \int_{\mathcal{F}_{g}} g(s) \mathrm{d} s
$$

respectively. We denote

$$
G_{\gamma}(t)=\gamma(t)^{-1} \int_{t}^{+\infty} g(s) \gamma(s) \mathrm{d} s
$$

Now, we are in a position to state our main result.

Theorem 2.2 ([23]) Let $\left(u_{0}, u_{1}\right) \in\left(V \bigcap H^{2}(\Omega)\right) \times V$, Assume that (2.1)-(2.2) hold and $\mathcal{R}_{g}<\frac{1}{2}$. If $G_{\gamma}(0)<\frac{(1-l)(2-l)}{2}$, then there exist positive constants $C$ and $v$ such that

$$
E(t) \leq C \gamma(t)^{-v}, \quad t \geq 0
$$

\section{Arbitrary Rate of Decay}

Now we define

$$
\Phi(t)=\frac{1}{\rho+1} \int_{\Omega}\left|u_{t}\right|^{\rho} u_{t} u \mathrm{~d} x+\int_{\Omega} \nabla u_{t} \cdot \nabla u \mathrm{~d} x+\frac{1}{2} \int_{\Gamma_{0}} p y^{2} \mathrm{~d} \Gamma+\int_{\Gamma_{0}} u y \mathrm{~d} \Gamma .
$$

Using (1.1) and (3.1), we have

$$
\begin{aligned}
\Phi^{\prime}(t)= & \frac{1}{\rho+1}\left\|u_{t}\right\|_{\rho+2}^{\rho+2}-\|\nabla u\|^{2}+\left\|\nabla u_{t}\right\|^{2}+\int_{\Omega} \nabla u \int_{0}^{t} g(t-s) \nabla u(s) \mathrm{d} s \mathrm{~d} x \\
& +\int_{\Omega} \Delta u_{t} u \mathrm{~d} \Gamma+2 \int_{\Gamma_{0}} u y_{t} \mathrm{~d} \Gamma-\int_{\Gamma_{0}} q(x) y^{2} \mathrm{~d} \Gamma .
\end{aligned}
$$

We use here the following identity due to [1], to give a better estimate for the term $\int_{\Omega} \nabla u \int_{0}^{t} g(t-s) \nabla u(s) \mathrm{d} s \mathrm{~d} x$ :

$$
\begin{aligned}
& \int_{\Omega} \nabla u \int_{0}^{t} g(t-s) \nabla u(s) \mathrm{d} s \mathrm{~d} x \\
& =\frac{1}{2}\left(\int_{0}^{t} g(s) \mathrm{d} s\right)\|\nabla u\|^{2}+\frac{1}{2} \int_{0}^{t} g(t-s)\|\nabla u(s)\|^{2} \mathrm{~d} s-\frac{1}{2}(g \circ \nabla u)(t) .
\end{aligned}
$$

From (2.1), (3.2) and (3.3), integration by parts and Young's inequality, we derive for any $\delta_{0}>0$,

$$
\begin{aligned}
\Phi^{\prime}(t) \leq & \frac{1}{\rho+1}\left\|u_{t}\right\|_{\rho+2}^{\rho+2}+\left(1+\delta_{0}\right)\left\|\nabla u_{t}(t)\right\|^{2}-\left(\frac{1+l}{2}-\delta_{0} \tilde{\lambda}\right)\|\nabla u\|^{2} \\
& +\frac{1}{2} \int_{0}^{t} g(t-s)\|\nabla u(s)\|^{2} \mathrm{~d} s-\frac{1}{2}(g \circ u)(t) \\
& +\frac{1}{\delta_{0}}\left\|y_{t}\right\|_{\Gamma_{0}}^{2}-\int_{\Gamma_{0}} q(x) y^{2} \mathrm{~d} \Gamma .
\end{aligned}
$$

As in [5], we have:

Lemma 3.1 For $u \in H_{0}^{1}(\Omega)$, we have 


$$
\int_{\Omega}\left(\int_{0}^{t} g(t-s)(u(t)-u(s)) \mathrm{d} s\right)^{2} \mathrm{~d} x \leq \lambda(1-l)(g \circ \nabla u)(t) .
$$

Now we define the functional

$$
\Psi(t)=\int_{\Omega}\left(\Delta u_{t}-\frac{1}{\rho+1}\left|u_{t}\right|^{\rho} u_{t}\right) \int_{0}^{t} g(t-s)(u(t)-u(s)) \mathrm{d} s \mathrm{~d} x .
$$

It follows from (1.1) and (3.6) that

$$
\begin{aligned}
\Psi^{\prime}(t)= & \int_{\Omega} \Delta u_{t} \int_{0}^{t} g^{\prime}(t-s)(u(t)-u(s)) \mathrm{d} s \mathrm{~d} x-\left(\int_{0}^{t} g(s) \mathrm{d} s\right)\left\|\nabla u_{t}\right\|^{2} \\
& +\left(1-\int_{0}^{t} g(s) \mathrm{d} s\right) \int_{\Omega} \nabla u(t) \cdot\left(\int_{0}^{t} g(t-s)(\nabla u(t)-\nabla u(s)) \mathrm{d} s\right) \mathrm{d} x \\
& +\int_{\Omega}\left(\int_{0}^{t} g(t-s)(\nabla u(t)-\nabla u(s)) \mathrm{d} s\right)^{2} \mathrm{~d} x-\frac{\int_{0}^{t} g(s) \mathrm{d} s}{\rho+1}\left\|u_{t}\right\|_{\rho+2}^{\rho+2} \\
& +\int_{\Omega} \nabla u_{t} \int_{0}^{t} g(t-s)(\nabla u(t)-\nabla u(s)) \mathrm{d} s \mathrm{~d} x \\
& -\int_{\Omega} \frac{1}{\rho+1}\left|u_{t}\right|^{\rho} u_{t} \int_{0}^{t} g^{\prime}(t-s)(u(t)-u(s)) \mathrm{d} s \mathrm{~d} x \\
& -\int_{\Gamma_{0}} y_{t}\left(\int_{0}^{t} g(t-s)(u(t)-u(s)) \mathrm{d} s\right) \mathrm{d} \Gamma \\
= & I_{1}-I_{2}+\left(1-\int_{0}^{t} g(s) \mathrm{d} s\right) I_{3}+I_{4}-I_{5}+I_{6}-I_{7}-I_{8} .
\end{aligned}
$$

For any $\delta>0$, we have

$$
I_{1} \leq \delta\left\|\nabla u_{t}(t)\right\|^{2}-\frac{g(0)}{4 \delta} \lambda\left(g^{\prime}(s) \circ \nabla u\right)(t) .
$$

For all measurable sets $\mathcal{A}$ and $\mathcal{F}$ such that $\mathcal{A}=\mathbb{R}^{+} \backslash \mathcal{F}, I_{3}, I_{4}$ and $I_{6}$ can be estimated as in [1]:

$$
\begin{aligned}
& I_{3} \leq \delta_{1}\|\nabla u\|^{2}+\frac{1-l}{4 \delta_{1}} \int_{\Omega} \int_{\mathcal{A}_{t}} g(t-s)|\nabla u(t)-\nabla u(s)|^{2} \mathrm{~d} s \mathrm{~d} x \\
&+\frac{3}{2}(1-l) \hat{g}(\mathcal{F})\|\nabla u\|^{2}+\frac{1}{2} \int_{\mathcal{F}_{t}} g(t-s)\|\nabla u(s)\|^{2} \mathrm{~d} s, \quad \delta_{1}>0, \\
& I_{4} \leq\left(1+\frac{1}{\delta_{2}}\right)(1-l) \int_{\Omega} \int_{\mathcal{A}_{t}} g(t-s)|\nabla u(t)-\nabla u(s)|^{2} \mathrm{~d} s \mathrm{~d} x \\
&+\left(1+\delta_{2}\right)(1-l) \hat{g}(\mathcal{F}) \int_{\Omega} \int_{\mathcal{F}_{t}} g(t-s)|\nabla u(t)-\nabla u(s)|^{2} \mathrm{~d} s \mathrm{~d} x, \quad \delta_{2}>0, \\
& I_{6} \leq \delta_{1}\left\|\nabla u_{t}\right\|^{2}+\frac{1}{4 \delta_{1}} \int_{\Omega} \int_{\mathcal{A}_{t}} g(t-s)|\nabla u(t)-\nabla u(s)|^{2} \mathrm{~d} s \mathrm{~d} x \\
& \quad+\frac{3}{2} \hat{g}(\mathcal{F})\left\|\nabla u_{t}\right\|^{2}+\frac{1}{2} \int_{\mathcal{F}_{t}} g(t-s)\|\nabla u(s)\|^{2} \mathrm{~d} s, \quad \delta_{1}>0,
\end{aligned}
$$

where $\hat{g}$ is defined in (2.8). For any $\delta>0$,

$$
I_{7} \leq \delta\left\|\nabla u_{t}(t)\right\|^{2}-\frac{g(0)}{4 \delta} \lambda\left(g^{\prime}(s) \circ \nabla u\right)(t) .
$$

For $I_{8}$, for $\delta_{3}, \delta_{4}>0$, we use a different estimate as 


$$
\begin{aligned}
I_{8}= & \int_{\Gamma_{0}} y_{t}\left(\int_{\mathcal{A}_{t}} g(t-s)(u(t)-u(s)) \mathrm{d} s\right) \mathrm{d} \Gamma \\
& +\int_{\Gamma_{0}} y_{t}\left(\int_{\mathcal{F}_{t}} g(t-s)(u(t)-u(s)) \mathrm{d} s\right) \mathrm{d} \Gamma \\
& \leq \frac{1}{2}\left\|y_{t}\right\|_{\Gamma_{0}}^{2}+\frac{\tilde{\lambda}(1-l)}{2} \int_{\Omega} \int_{\mathcal{A}_{t}} g(t-s)|\nabla u(t)-\nabla u(s)|^{2} \mathrm{~d} s \mathrm{~d} x \\
& +\frac{1}{4 \delta_{3}} \hat{g}(\mathcal{F})\left\|y_{t}\right\|_{\Gamma_{0}}^{2} \\
& +\delta_{3} \tilde{\lambda} \hat{g}(\mathcal{F})\|\nabla u\|^{2}+\frac{1}{4 \delta_{4}}\left\|y_{t}\right\|_{\Gamma_{0}}^{2} \\
& +\delta_{4} \tilde{\lambda}(1-l) \int_{\mathcal{F}_{t}} g(t-s)\|\nabla u(s)\|^{2} \mathrm{~d} s .
\end{aligned}
$$

Taking into account these estimates in (3.6), let $t_{*}$ be a number such that $\int_{0}^{t_{*}} g(s) \mathrm{d} s=g_{*}$, we obtain that

$$
\begin{aligned}
\Psi^{\prime}(t) \leq & \left(-\frac{g_{*}}{2}+\delta_{1}\right)\left\|\nabla u_{t}\right\|^{2}-\frac{g_{*}}{\rho+1}\left\|u_{t}\right\|_{\rho+2}^{\rho+2} \\
+ & \left\{\left(1-g_{*}\right)\left(\delta_{1}+\frac{3}{2}(1-l) \hat{g}(\mathcal{F})\right)+\delta_{3} \tilde{\lambda} \hat{g}(\mathcal{F})+\delta\right\} \\
& \times\|\nabla u\|^{2}(1-l)\left(\frac{1-g_{*}}{4 \delta_{1}}+\frac{1+\delta_{2}}{\delta_{2}}+\frac{\tilde{\lambda}}{2}\right) \int_{\Omega} \int_{\mathcal{A}_{t}} g(t-s)|\nabla u(t)-\nabla u(s)|^{2} \mathrm{~d} s \mathrm{~d} x \\
& -\frac{3}{4 \delta} g(0) \lambda\left(g^{\prime} \circ \nabla u\right)(t) \\
+ & \left(1+\delta_{2}\right)(1-l) \hat{g}(\mathcal{F}) \int_{\Omega} \int_{\mathcal{F}_{t}} g(t-s)|\nabla u(t)-\nabla u(s)|^{2} \mathrm{~d} s \mathrm{~d} x \\
& +\left(\frac{1-g_{*}}{2}+\delta_{4} \tilde{\lambda}(1-l)\right) \int_{\mathcal{F}_{t}} g(t-s)\|\nabla u(s)\|^{2} \\
& +\left(\frac{1}{2}+\frac{\hat{g}(\mathcal{F})}{4 \delta_{3}}+\frac{1}{4 \delta_{4}}\right)\left\|y_{t}\right\|_{\Gamma_{0}}^{2} .
\end{aligned}
$$

Let

$$
I(t)=\int_{\Omega} \int_{0}^{t} G_{\gamma}(t-s)|\nabla u(s)|^{2} \mathrm{~d} s \mathrm{~d} x,
$$

and $G_{\gamma}(t)$ is given in (2.11), we define the following functional

$$
F(t)=M E(t)+\varepsilon \Phi(t)+\Psi(t)+\epsilon I(t)
$$

then we know from [1] that

$$
\begin{aligned}
I^{\prime}(t) \leq & G_{\gamma}(0)\|\nabla u\|^{2}-\eta(t) \int_{0}^{t} G_{\gamma}(t-s)\|\nabla u(s)\|^{2} \mathrm{~d} s \\
& -\int_{0}^{t} g(t-s)\|\nabla u(s)\|^{2} \mathrm{~d} s .
\end{aligned}
$$

At the same time, we have the following lemmas.

Lemma 3.2 For $M$ large enough, there exist two positive constants $\rho_{1}$ and $\rho_{2}$ such that

$$
\rho_{1}(E(t)+I(t)) \leq F(t) \leq \rho_{2}(E(t)+I(t)) .
$$

Proof. See, e.g. Liu [5].

Proof of Theorem 2.2 By using (2.7), (3.4), (3.13)-(3.16), a series of com- 
putations yields, for $t \geq t_{*}$,

$$
\begin{aligned}
F^{\prime}(t) \leq & \left(\frac{M}{2}-\frac{3}{4 \delta} g(0) \lambda\right)\left(g^{\prime} \circ \nabla u\right)(t)-\left(\frac{g_{*}}{\rho+1}-\frac{\varepsilon}{1+\rho}\right)\left\|u_{t}(t)\right\|_{\rho+2}^{\rho+2} \\
& -\left[\frac{M}{2}+\frac{g_{*}}{2}-\delta_{1}-\varepsilon\left(1+\delta_{0}\right)\right]\left\|\nabla u_{t}(t)\right\|^{2} \\
& +\left(1+\delta_{2}\right)(1-l) \hat{g}(\mathcal{F}) \int_{\Omega} \int_{\mathcal{F}_{t}} g(t-s)|\nabla u(t)-\nabla u(s)|^{2} \mathrm{~d} s \mathrm{~d} x \\
& +\left\{\left(1-g_{*}\right)\left(\delta_{1}+\frac{3}{2}(1-l) \hat{g}(\mathcal{F})\right)+\delta_{3} \tilde{\lambda} \hat{g}(\mathcal{F})+\delta+e G_{\gamma}(0)-\varepsilon\left(\frac{1+l}{2}-\delta_{0} \tilde{\lambda}\right)\right\}\|\nabla u\|^{2} \\
& +(1-l)\left(\frac{1-g_{*}}{4 \delta_{1}}+\frac{1+\delta_{2}}{\delta_{2}}+\frac{\tilde{\lambda}}{2}\right) \int_{\Omega} \int_{\mathcal{A}_{t}} g(t-s)|\nabla u(t)-\nabla u(s)|^{2} \mathrm{~d} s \mathrm{~d} x \\
& -\left(\epsilon-\frac{\varepsilon}{2}\right) \int_{0}^{t} g(t-s)\|\nabla u(s)\|^{2} \mathrm{~d} s-\frac{\varepsilon}{2}(g \circ \nabla u)(t)\left(\frac{1-g_{*}}{2}+\delta_{4} \tilde{\lambda}(1-l)\right) \int_{\mathcal{F}_{t}} g(t-s)\|\nabla u(s)\|^{2} \mathrm{~d} s \mathrm{~d} x \\
& -\epsilon \eta(t)-\varepsilon \int_{\Gamma_{0}} q(x) y^{2} \mathrm{~d} \Gamma-\left[M p_{0}-\frac{\varepsilon}{\delta_{0}}-\left(\frac{1}{2}+\frac{\hat{g}(\mathcal{F})}{4 \delta_{3}}+\frac{1}{4 \delta_{4}}\right)\right]\left\|y_{t}\right\|_{\Gamma_{0}}^{2} \cdot
\end{aligned}
$$

For $n \in \mathbb{N}$, as in [32] we introduce the sets

$$
\mathcal{A}_{n}=\left\{s \in \mathbb{R}^{+}: n g^{\prime}(s)+g(s) \leq 0\right\} .
$$

It is easy to see that

$$
\bigcup_{n} \mathcal{A}_{n}=\mathbb{R}^{+} \backslash\left\{\mathcal{F}_{g} \cup \mathcal{N}_{g}\right\}
$$

where $\mathcal{F}_{g}$ is given in (2.9) and $\mathcal{N}_{g}$ is the null set where $g^{\prime}$ is not defined. Additionally, we denote $\mathcal{F}_{n}=\mathbb{R}^{+} \backslash \mathcal{A}_{n}$, then

$$
\lim _{n \rightarrow \infty} \hat{g}\left(\mathcal{F}_{n}\right)=\hat{g}\left(\mathcal{F}_{g}\right) \text {, }
$$

since $\mathcal{F}_{n+1} \subset \mathcal{F}_{n}$ for all $n$ and $\bigcap_{n} \mathcal{F}_{n}=\mathcal{F}_{g} \cup \mathcal{N}_{g}$. Then, we take $\mathcal{A}=\mathcal{A}_{n}$ and $\mathcal{F}=\mathcal{F}_{n}$ in (3.18), it follows that

$$
\begin{aligned}
F^{\prime}(t) \leq & \left(\frac{M}{2}-\frac{3}{4 \delta} g(0) \lambda\right)\left(g^{\prime} \circ \nabla u\right)(t)-\left(\frac{g_{*}}{\rho+1}-\frac{\varepsilon}{\rho+1}\right)\left\|u_{t}(t)\right\|_{\rho+2}^{\rho+2} \\
& -\left[\frac{M}{2}+\frac{g_{*}}{2}-\delta_{1}-\varepsilon\left(1+\delta_{0}\right)\right]\left\|\nabla u_{t}(t)\right\|^{2} \\
& -\left(\epsilon-\frac{1+\varepsilon-g_{*}}{2}-\delta_{4} \tilde{\lambda}(1-l)\right) \int_{0}^{t} g(t-s)\|\nabla u(s)\|^{2} \mathrm{~d} s \\
& +\left\{\left(1-g_{*}\right)\left(\delta_{1}+\frac{3}{2}(1-l) \hat{g}\left(\mathcal{F}_{n}\right)\right)+\delta_{3} \tilde{\lambda} \hat{g}\left(\mathcal{F}_{n}\right)+\delta+e G_{\gamma}(0)\right. \\
& \left.-[\sigma+(1-\sigma)] \varepsilon \frac{1+l}{2}+\varepsilon \delta_{0} \tilde{\lambda}\right\}\|\nabla u\|^{2} \\
& -(1-l)\left(\frac{1-g_{*}}{4 \delta_{1}}+\frac{1+\delta_{2}}{\delta_{2}}+\frac{\tilde{\lambda}}{2}\right) \int_{\Omega} \int_{\mathcal{A}_{t}} g(t-s)|\nabla u(t)-\nabla u(s)|^{2} \mathrm{~d} s \mathrm{~d} x \\
& -\epsilon \eta(t) I(t)-\varepsilon \int_{\Gamma_{0}} q(x) y^{2} \mathrm{~d} \Gamma-\left[M p_{0}-\frac{\varepsilon}{\delta_{0}}-\left(\frac{1}{2}+\frac{\hat{g}\left(\mathcal{F}_{n}\right)}{4 \delta_{3}}+\frac{1}{4 \delta_{4}}\right)\right]\left\|y_{t}\right\|_{\Gamma_{0}}^{2},
\end{aligned}
$$


for some $0<\delta<1$. Since $\mathcal{R}_{g}=\hat{g}\left(\mathcal{F}_{g}\right)<\frac{1}{2}$, we can choose $\varepsilon, \delta_{2}$ small enough and $n, t_{*}$ large enough such that

$$
\frac{\varepsilon}{2}-\left(1+\delta_{2}\right)(1-l) \hat{g}\left(\mathcal{F}_{n}\right) \geq 0
$$

and

$$
\frac{3}{2}(1-l)\left(1-g_{*}\right) \hat{g}\left(\mathcal{F}_{n}\right)-\sigma \varepsilon \frac{1+l}{2}<0
$$

with $\sigma=\frac{3(1-l)\left(1-g_{*}\right)}{2 g_{*}(1+l)}$. Note that for $t_{*}$ large enough. Furthermore, we require that

$$
\begin{aligned}
\frac{1+\varepsilon-g_{*}}{2}+\delta_{4} \tilde{\lambda}(1-l) \leq \epsilon \leq & \frac{1}{G_{\gamma}(0)}\left((1-\sigma) \varepsilon \frac{1+l}{2}\right. \\
& \left.\quad-\left(1-g_{*}\right) \delta_{1}-\delta_{3} \tilde{\lambda} \hat{g}\left(\mathcal{F}_{n}\right)-\varepsilon \delta_{0} \tilde{\lambda}+\delta\right) .
\end{aligned}
$$

Combining (3.24) and (3.25), we obtain

$$
\left(1-g_{*}\right)\left(\delta_{1}+\frac{3}{2}(1-l) \hat{g}\left(\mathcal{F}_{n}\right)\right)+\delta_{3} \tilde{\lambda} \hat{g}\left(\mathcal{F}_{n}\right)+\delta+e G_{\gamma}(0)-\varepsilon \frac{1+l}{2}+\varepsilon \delta_{0} \tilde{\lambda}<0
$$

Choose our constants properly so that:

$$
\begin{gathered}
\frac{M}{2}-\frac{3}{4 \delta} g(0) \lambda \geq \frac{M}{4}, \\
M p_{0}-\frac{\varepsilon}{\delta_{0}}-\left(\frac{1}{2}+\frac{\hat{g}\left(\mathcal{F}_{n}\right)}{4 \delta_{3}}+\frac{1}{4 \delta_{4}}\right) \geq 0, \\
(1-l)\left(\frac{1-g_{*}}{4 \delta_{1}}+\frac{1+\delta_{2}}{\delta_{2}}+\frac{\tilde{\lambda}}{2}\right)-\frac{M}{4 n}<0
\end{gathered}
$$

together with (3.22) yield

$$
F^{\prime}(t) \leq-C_{1} E(t)-\epsilon \eta(t) I(t), \quad t \geq t_{*} .
$$

As $\eta(t)$ is decreasing, we have $\eta(t) \leq \eta(0)$ for all $t \geq t_{*}$. Then (3.30) becomes

$$
F^{\prime}(t) \leq-\frac{C_{1}}{\eta(0)} \eta(t) E(t)-\epsilon \eta(t) I(t), \quad t \geq t_{*} .
$$

Since $F(t)$ is equipped with $E(t)+I(t)$, we get

$$
F^{\prime}(t) \leq-C_{2} \eta(t) F(t),
$$

integrating (3.31) over $\left[t_{*}, t\right]$ yields

$$
F(t) \leq e^{-C_{2} \int_{t_{*}}^{t} \eta(s) d s} F\left(t_{*}\right), \quad t \geq t_{*} .
$$

Then using the left hand side inequality in (3.17), we get

$$
\rho_{1}(E(t)+I(t)) \leq e^{-C_{2} \int_{t_{*}}^{t} \eta(s) \mathrm{d} s} F\left(t_{*}\right), \quad t \geq t_{*} .
$$


By virtue of the continuity and boundedness of $E(t)$ in the interval $\left[0, t_{*}\right]$, we conclude that

$$
E(t) \leq C \gamma^{-v}(t), \quad t \geq 0
$$

for some positive constants $C$ and $v$.

\section{Acknowledgements}

This work was in part supported by Shanghai Second Polytechnical University and the key discipline "Applied Mathematics" of Shanghai Second Polytechnic University with contract number XXKPY1604.

\section{References}

[1] Tatar, N. (2011) Arbitrary Decays in Linear Viscoelasticity. Journal of Mathematical Physics, 52, Article ID: 013502.

[2] Cavalcanti, M.M., Domingos Cavalcanti, V.N. and Ferreira, J. (2001) Existence and Uniform Decay for a Non-Linear Viscoelastic Equation with Strong Damping. Mathematical Methods in the Applied Sciences, 24, 1043-1053. https://doi.org/10.1002/mma.250

[3] Love, A.E.H. (1944) A Treatise on the Mathematical Theory of Elasticity. Dover, New York.

[4] Fabrizio, M. and Morro, A. (1992) Mathematical Problems in Linear Viscoelasticity. SIAM Studies in Applied Mathematics, No. 12, Philadelphia.

[5] Liu, W. (2014) Arbitrary Rate of Decay for a Viscoelastic Equation with Acoustic Boundary Conditions. Applied Mathematics Letters, 38, 155-161. https://doi.org/10.1090/S0002-9904-1974-13714-6

[6] Beale, J.T. and Rosencrans, S.I. (1974) Acoustic Boundary Conditions. Bulletin of the AMS, 80, 1276-1278.

[7] Frota, C.L. and Goldstein, J.A. (2000) Some Nonlinear Wave Equations with Acoustic Boundary Conditions. Journal of Differential Equations, 164, 92-109. https://doi.org/10.1006/jdeq.1999.3743

[8] Frota, C.L. and Larkin, N.A. (2006) Uniform Stabilization for a Hyperbolic Equation with Acoustic Boundary Conditions in Simple Connected Domains. In: Cazenave, T., Costa, D., Lopes, O., Manásevich, R., Rabinowitz, P., Ruf, B. and Tomei, C., Eds., Contributions to Nonlinear Analysis, Vol. 66, Birkhauser, Basel, 297-312. https://doi.org/10.1007/3-7643-7401-2_20

[9] Park, J.Y. and Park, S.H. (2011) Decay Rate Estimates for Wave Equations of Memory Type with Acoustic Boundary Conditions. Nonlinear Analysis, 74, 993-998.

[10] Munoz Rivera, J.E. and Qin, Y. (2003) Polynomial Decay for the Energy with an Acoustic Boundary Condition. Applied Mathematics Letters, 16, 249-256.

[11] Dafermos, C.M. (1970) Asymptotic Stability in Viscoelasticity. Archive for Rational Mechanics and Analysis, 37, 297-308. https://doi.org/10.1007/BF00251609

[12] Dafermos, C.M. (1970) An Abstract Volterra Equation with Applications to Linear Viscoelasticity. Journal of Differential Equations, 7, 554-569.

[13] Berrimi, S. and Messaoudi, S.A. (2006) Existence and Decay of Solutions of a Viscoelastic Equation with a Nonlinear Source. Nonlinear Analysis, 64, 2314-2331.

[14] Cavalcanti, M.M., Domingos Cavalcanti, V.N. and Martinez, P. (2008) General Decay Rate Estimates for Viscoelastic Dissipative Systems. Nonlinear Analysis, 68, 177-193. 
[15] Liu, W.J. and Sun, Y. (2014) General Decay of Solutions for a Weak Viscoelastic Equation with Acoustic Boundary Conditions. Zeitschrift für Angewandte Mathematik und Physik, 65, 125-134. https://doi.org/10.1007/s00033-013-0328-y

[16] Messaoudi, S.A. and Tatar, N.-E. (2007) Global Existence and Uniform Stability of Solutions for a Quasilinear Viscoelastic Problem. Mathematical Methods in the Applied Sciences, 30, 665-680. https://doi.org/10.1002/mma.804

[17] Messaoudi, S.A. and Tatar, N.-E. (2009) Exponential Decay for a Quasilinear Viscoelastic Equation. Mathematische Nachrichten, 282, 1443-1450. https://doi.org/10.1002/mana.200610800

[18] Messaoudi, S.A. (2008) General Decay of Solutions of a Viscoelastic Equation. Journal of Mathematical Analysis and Applications, 341, 1457-1467.

[19] Wu, S.T. (2012) General Decay of Energy for a Viscoelastic Equation with Damping and Source Terms. Taiwanese Journal of Mathematics, 16, 113-128.

[20] Han, X. and Wang, M. (2009) General Decay of Energy for a Viscoelastic Equation with Nonlinear Damping. Mathematical Methods in the Applied Sciences, 32, 346358. https://doi.org/10.1002/mma.1041

[21] Park, J.Y. and Park, S.H. (2009) General Decay for Quasiliear Viscoelastic Equations with Nonlinear Weak Damping. Journal of Mathematical Physics, 50, 1-10. https://doi.org/10.1063/1.3187780

[22] Fabrizio, M. and Polidoro, S. (2002) Asymptotic Decay for Some Differential Systems with Fading Memory. Applicable Analysis, 81, 1245-1264. https://doi.org/10.1080/0003681021000035588

[23] Tatar, N. (2008) Polynomial Stability without Polynomial Decay of the Relaxation Function. Mathematical Methods in the Applied Sciences, 31, 1874-1886. https://doi.org/10.1002/mma.1018

[24] Yu, S.Q. (2009) Polynomial Stability of Solutions for a System of Non-Linear Viscoelastic Equations. Applicable Analysis, 88, 1039-1051. https://doi.org/10.1080/00036810903114825

[25] Araújo, R.O., Ma, T. and Qin, Y. (2013) Long-Time Behavior of a Quasilinear Viscoelastic Equation with Past History. Journal of Differential Equations, 254, 40664087.

[26] Cavalcanti, M.M., Domingos Cavalcanti, V.N., Prates Filho, J.A. and Soriano, J.A. (2001) Existence and Uniform Decay Rates for Viscoelastic Problems with Non-Linear Boundary Damping. Differential and Integral Equations, 14, 85-116.

[27] Chueshov, I. and Lasiecka, I. (2008) Long-Time Behavior of Second Order Evolution Equations with Nonlinear Damping. Vol. 195, Memoirs of the American Mathematical Society, Providence.

[28] Munoz Rivera, J.E., Lapa, E.C. and Barreto, R. (1996) Decay Rates for Viscoelastic Plates with Memory. Journal of Elasticity, 44, 61-87. https://doi.org/10.1007/BF00042192

[29] Wu, S.T. (2011) General Decay of Solutions for a Viscoelastic Equation with Nonlinear Damping and Source Terms. Acta Mathematica Scientia, 31, 1436-1448.

[30] Han, X. and Wang, M. (2009) Global Existence and Uniform Decay for a Nonlinar Viscoelastic Equation with Damping. Nonlinear Analysis, 70, 3090-3098.

[31] Qin, Y., Feng, B. and Zhang, M. (2014) Uniform Attractors for a Non-Autonomous Viscoelastic Equation with a Past History. Nonlinear Analysis, 101, 1-15.

[32] Pata, V. (2006) Exponential Stability in Linear Viscoelasticity. Quarterly of Applied Mathematics, 6, 499-513. https://doi.org/10.1090/S0033-569X-06-01010-4 
Submit or recommend next manuscript to SCIRP and we will provide best service for you:

Accepting pre-submission inquiries through Email, Facebook, LinkedIn, Twitter, etc. A wide selection of journals (inclusive of 9 subjects, more than 200 journals)

Providing 24-hour high-quality service

User-friendly online submission system

Fair and swift peer-review system

Efficient typesetting and proofreading procedure

Display of the result of downloads and visits, as well as the number of cited articles Maximum dissemination of your research work

Submit your manuscript at: http://papersubmission.scirp.org/

Or contact jamp@scirp.org 